\title{
EFFECTS OF DRY FRICTION DAMPING ON THE OCCURRENCE OF LOCALIZED FORCED VIBRATIONS IN NEARLY CYCLIC STRUCTURES
}

\author{
S.-T. Wei and C. Pierre \\ Department of Mechanical Engineering and Applied Mechanics, 3200 G. G. Brown Building, \\ The University of Michigan, Ann Arbor, Michigan 48109-2125, U.S.A.
}

(Received 14 April 1988, and in revised form 21 August 1988)

\begin{abstract}
A multi-harmonic balance method is applied to analyze the occurrence of strongly localized forced vibrations in a mistuned cyclic assembly of coupled, non-linear component structures under traveling wave excitation. In addition to considering the effects of the strength of coupling between and disorder among component systems, the study also investigates the relative effects of dry friction damping and viscous damping on the occurrence of strong localization. Results indicate that the degree of localization decreases as the amount of damping increases and that dry friction damped systems are more susceptible to localized vibrations than viscously damped ones. This multi-degree-offreedom system with many dry friction dampers is analyzed by a multi-harmonic frequency domain procedure. The issues of accuracy and convergence of the method when applied to systems with several strongly non-linear elements are discussed. This frequency domain solution technique is compared to time domain methods.
\end{abstract}

\section{INTRODUCTION}

In structural dynamics, the sensitivity to disorder is a major concern in the design and analysis of nominally periodic structures made of coupled component systems. It is now well known that the ratio of internal coupling strength to disorder strength is the key parameter governing the sensitivity to disorder. For small values of this ratio, small structural irregularities, which break the structure's periodicity, may inhibit the propagation of vibration from the source of excitation and confine the vibration modes to a narrow geometric region-a phenomenon known as normal mode localization.

A number of studies have provided analyses of localized free vibrations for a variety of nearly periodic structures such as chains of coupled pendulums [1-3], coupled beam assemblies [4], bladed-disk assemblies [5], and cyclic assemblies [6-8]. All these studies led to the conclusion that the degree of localization of the mode shapes depends primarily upon the ratio of coupling to disorder. Nonetheless, besides coupling and disorder, the magnitude and type of damping and the type of excitation must be considered in the study of localized forced vibrations. Hodges and Woodhouse [2] and Pierre [4] investigated localized forced vibrations under a local source of excitation for chains of coupled pendulums and coupled beam assemblies, respectively. Kissel [9] also studied wave propagation and weak localization in disordered spring-mass chains, using a statistical approach. Wei and Pierre [7] demonstrated the occurrence of localized forced vibrations in mistuned assemblies with cyclic symmetry when all component systems are excited harmonically. However, the role of damping on the strength of localized forced vibrations has not been explored. Therefore, the primary objective of this paper is to investigate the effects of dry friction and viscous damping on the degree of forced vibration localization. 
To that purpose, the cyclic assembly of coupled component systems chosen recently by Wei and Pierre [7] is investigated, with both friction damping and viscous damping present in the system.

Because of the piecewise type non-linearity of friction elements, previous studies of the frequency responses of cyclic structures with multiple dry friction dampers have been restricted to one-harmonic approximations. In several studies the single-harmonic balance method has shown adequate accuracy for the parameter ranges investigated, but the region of acceptability has not yet been justified. In the parameter region where the one-harmonic approach cannot provide satisfactory results (e.g., low excitation frequency or large dry friction force-that is, when stick-slip motions occur), a more precise method must be used such as time domain numerical integration. Although numerical integration methods generate accurate results, the time step and the duration of the integration vary as the system parameters change. Obtaining the steady state responses of multiple degree-of-freedom (DOF) systems with low damping ratios can also be computationally very expensive. Therefore, it is often difficult and impractical to conduct frequency domain analyses and automatic parametric studies through time integration. A compromise (with reasonable computing time and adequate accuracy) may be reached by choosing a multi-harmonic frequency domain analysis. Lau et al. [10] developed such a multiharmonic technique, the so-called incremental harmonic balance (IHB) method, to study systems with cubic non-linearities. Pierre and Dowell [11] applied the IHB method to analyze the dynamic instability of plates. This method was also modified by Pierre et al. [12] to analyze one- and two-DOF dry friction damped systems. Another frequency domain analysis of non-linear systems, a Galerkin/Newton-Raphson (GNR) method, was further developed by Ferri and Dowell [13] to handle a beam structure with two discrete dry friction elements attached to it. Ferri [14] showed that this GNR method was equivalent to the IHB method. However, the GNR procedure appears to provide a more automatic algorithm and to be more suitable to systems with several DOF. Therefore, in the present study, the GNR method is applied to obtain the multi-harmonic frequency response of the system. It is worth noting that previous studies of dry friction damped systems were restricted either to a single-harmonic analysis of many dry friction elements or to a multi-harmonic analysis of one, or at most two, dry friction dampers. Here, a multiple harmonic analysis of many dry friction elements is carried out. Note that a so-called fast Galerkin method recently developed by Ling and $W u$ [15] reduces the computation time required to obtain the higher harmonic terms. The method is thought to be essentially an efficient implementation of the IHB (or GNR) method.

Three major issues are addressed in this study. First, the results of the GNR analysis are compared with time integration results, and the acceptability of the one-harmonic approximation is discussed in terms of the system parameters. Second, the combined effects of disorder, coupling, dry friction damping and viscous damping on the forced response of a cyclic structure are explored. Finally, the occurrence of localized forced vibrations in weakly coupled mistuned assemblies is studied, and the relative effects of dry friction and viscous damping on the degree of localization are analyzed. This is believed to be the first study of localization for engineering structures with non-linearities.

\section{EQUATIONS OF MOTION}

The nearly cyclic assembly of coupled component systems shown in Figure 1 is studied. Each subsystem, consisting of a single-DOF oscillator with both dry friction damping and viscous damping, is coupled to the adjacent component systems through linear springs. The same system without dry friction dampers has been recently used by Wei 


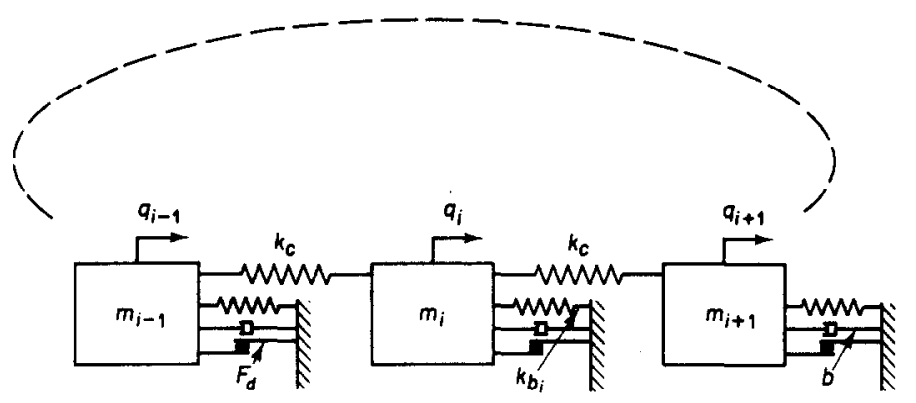

Figure 1. Geometry of nearly periodic structure with cyclic symmetry.

and Pierre $[6,7]$ as a simple model of continuously shrouded bladed disk assemblies to study localized free and forced vibrations in mistuned assemblies. The dry friction damping introduced here may be regarded as a model for platform damping in a turbine blade system. By component mode analysis it can be shown that each single-DOF component system is a one-structural mode approximation of a beam-like structure with a dry friction damper attached to the ground. Although the multi-DOF per blade model [16-18] and flexible dry friction damper model [19-22] allow greater flexibility in modeling the practical system, to reach the objectives of the present study only a single-DOF oscillator and a Coulomb's dry friction damper (perfectly rigid when the damper is stuck) are adopted. The obvious limitation of this model is that it can only be used to predict the response of the system when slip modes are dominant. Therefore, it cannot be applied to design the optimal dry friction force for the dry friction damped system. To achieve this goal a multi-DOF per blade model (or a flexible damper, or both) ought to be adopted instead.

For simplicity, disorder, or mistuning, is assumed to originate from discrepancies in the oscillators' stiffnesses. Also, the static and dynamic coefficients of friction are assumed equal. Therefore, the equations of motion of the system are

$$
\ddot{q}_{i}+2 \zeta \omega_{b} \dot{q}_{i}+\omega_{b i}^{2} q_{i}+2 \omega_{c}^{2} q_{i}-\omega_{c}^{2} q_{i-1}-\omega_{c}^{2} q_{i+1}+\left(F_{d} / m\right) \operatorname{Sgn}\left(\dot{q}_{i}\right)=F_{i} / m, \quad i=1, \ldots, N,
$$

where $q_{0} \equiv q_{N}$ and $q_{N+1} \equiv q_{1}$ (a list of nomenclature is given in Appendix II). The external force for a given engine order excitation, $n$, is harmonic in time with phase differences between component systems. This type of excitation has been widely used in the literature for bladed-disk assemblies [20]. One has

$$
F_{i}=F \cos \left(\omega t+\phi_{i}\right), \quad i=1, \ldots, N,
$$

where

$$
\phi_{i}=2 \pi n(i-1) / N, \quad i=1, \ldots, N .
$$

Upon introducing the following non-dimensional quantities,

$$
\begin{gathered}
\Delta f_{i}=\frac{\omega_{b i}^{2}-\omega_{b}^{2}}{\omega_{b}^{2}}, \quad x_{i}=\frac{m \omega_{b}^{2} q_{i}}{F_{d}}, \quad i=1, \ldots, N, \quad R^{2}=\omega_{c}^{2} / \omega_{b}^{2}, \\
\rho=F / F_{d}, \quad \Omega=\omega / \omega_{b}, \quad \tau=\omega t,
\end{gathered}
$$

where $\omega_{b}$ is the individual oscillators' nominal natural frequency and $\omega_{c}$ is the coupling 
frequency, equation (1) becomes

$$
\begin{aligned}
\Omega^{2} x_{i}^{\prime \prime} & +2 \zeta \Omega x_{i}^{\prime}+\left(1+\Delta f_{i}+2 R^{2}\right) x_{i}-R^{2} x_{i+1}-R^{2} x_{i-1}+\operatorname{Sgn}\left(x_{i}^{\prime}\right) \\
& =\rho \cos \left(\tau+\phi_{i}\right), \quad i=1, \ldots, N .
\end{aligned}
$$

The three key dimensionless parameters in equation (5) are $\Delta f_{i}, R^{2}$ and $\rho . \Delta f_{i}$ is the disorder (or mistuning) strength for the $i$ th subsystem, $R^{2}$ is the coupling strength, and $\rho$ is the ratio of external force to dry friction force. A multi-harmonic balance analysis is chosen to solve this set of non-linear equations.

\section{METHOD OF ANALYSIS: GNR PROCEDURE}

The Galerkin/Newton-Raphson method (GNR) developed by Ferri and Dowell [13] is outlined below and applied to obtain the steady state response of the multi-DOF and multi-dry function damper system described by equation (5).

Equation (5) can be written in matrix form as

$$
\Omega^{2}[M] \mathbf{x}^{\prime \prime}+\Omega[C] \mathbf{x}^{\prime}+[K] \mathbf{x}+\mathbf{G}\left(\mathbf{x}^{\prime}\right)=\mathbf{0},
$$

where $[M]=[I],[C]=2 \zeta[I]$,

$$
[K]=\left[\begin{array}{cccccc}
1+2 R^{2}+\Delta f_{1} & -R^{2} & 0 & \cdots & 0 & -R^{2} \\
-R^{2} & 1+2 R^{2}+\Delta f_{2} & -R^{2} & 0 & \cdots & 0 \\
0 & \ddots & \ddots & \ddots & \ddots & \vdots \\
\vdots & \ddots & \ddots & \ddots & \ddots & 0 \\
0 & \ddots & \ddots & \ddots & \ddots & -R^{2} \\
-R^{2} & 0 & \cdots & 0 & -R^{2} & 1+2 R^{2}+\Delta f_{N}
\end{array}\right]
$$

is a "nearly cyclic" matrix, and

$$
\mathbf{G}\left(\mathbf{x}^{\prime}\right)=\left\{G_{1}, G_{2}, \ldots, G_{N}\right\}^{\mathrm{T}}, \quad G_{i}=\operatorname{Sgn}\left(x_{i}^{\prime}\right)-\rho \cos \left(\tau+\phi_{i}\right), \quad i=1, \ldots, N,
$$

is the vector of non-linear and external force terms.

An approximate, periodic of period $2 \pi$, multi-harmonic solution is assumed,

$$
\mathbf{x} \simeq \hat{\mathbf{x}}=\sum_{j=1,3}^{2 N H-1} \mathbf{x}^{c j} \cos j \tau+\mathbf{x}^{s j} \sin j \tau .
$$

Since equation (9) cannot exactly satisfy equation (6), the Galerkin procedure is applied by substituting the expansion (9) into the governing equation (6) and requiring the resulting residue to be orthogonal to the space spanned by $(\cos j \tau, \sin j \tau)_{j=1,3, \ldots,(2 N H-1)}$. This yields

$$
\int_{0}^{2 \pi}\left[\Omega^{2}[M] \hat{\mathbf{x}}^{\prime \prime}+\Omega[C] \hat{\mathbf{x}}^{\prime}+[K] \hat{\mathbf{x}}+\mathbf{G}\left(\hat{\mathbf{x}}^{\prime}\right)\right] \quad\left(\begin{array}{c}
\cos \tau \\
\cos 3 \tau \\
\vdots \\
\cos (2 N H-1) \tau \\
\sin \tau \\
\sin 3 \tau \\
\vdots \\
\sin (2 N H-1) \tau
\end{array}\right)^{\mathrm{T}}=[0],
$$

where [0] is a $2 N H$ by $N$ null matrix. It is worth noting that the even harmonic terms can be shown to be identically zero by considering equation (5). Equation (10) can be 
rearranged in vector form as

$$
\mathbf{R}(\mathbf{y})=\mathbf{0}
$$

where

$$
y=\left\{x_{1}^{c 1}, \ldots, x_{1}^{c(2 N H-1)}, x_{1}^{s 1}, \ldots, x_{1}^{s(2 N H-1)}, x_{2}^{c 1}, \ldots, x_{N}^{s(2 N H-1)}\right\}^{\mathrm{T}}
$$

is a $(2 \cdot N H \cdot N)$ dimensional vector, and $\mathbf{R}$ is a residue vector of dimension $(2 \cdot N H \cdot N)$ given in Appendix I.

The Newton-Raphson iteration procedure is used to solve the above system of $(2 \cdot N H \cdot N)$ non-linear algebraic equations. From an initial estimate $\mathrm{y}_{0}$, the increment $\Delta y$ is given by

$$
\Delta \mathbf{y}=-\partial \mathbf{R} /\left.\partial \mathbf{y}\right|_{0} ^{-1} \mathbf{R}\left(\mathbf{y}_{0}\right),
$$

where $\partial \mathbf{R} /\left.\partial \mathbf{y}\right|_{0}$ is a $(2 \cdot N H \cdot N)$ by $(2 \cdot N H \cdot N)$ matrix given in Appendix I. The new estimate for $\mathbf{y}$ is therefore given by

$$
\mathbf{y}^{N E W}=\mathbf{y}^{O L D}+\Delta \mathbf{y}
$$

Equations (13) and (14) are iterated until the residue vector $\mathbf{R}(\mathbf{y})$ is approximately equal to zero and the vector $\Delta y$ is within the required accuracy; then convergence for the solution $\mathbf{y}$ of equation (11) is reached.

Two types of algorithms were adopted to calculate the integrals involving the sign function and its derivative. The first one consisted of a closed form evaluation by using the exact sign function and its derivative (twice the Dirac delta function), while the other approximated the sign function by a continuous function. In the present study, the former (described by Pierre et al. [12]) required less computing time, while the latter provided better convergence of the residue vector. Therefore, the exact sign function was usually chosen, except in parameter regions that led to convergence difficulties, where the alternative approach was applied and the following approximate function was used:

$$
\operatorname{Sgn}\left(x_{i}^{\prime}\right) \simeq(2 / \pi) \tan ^{-1}\left(\gamma x_{i}^{\prime}\right), \quad i=1, \ldots, N, \quad \text { for } \gamma \gg 1,
$$

where $\gamma$ determines the level of approximation of the sign function. As $\gamma$ approaches infinity, this approximate continuous function tends to the exact sign function and its derivative to twice the Dirac impulse function. However, choosing $\gamma$ very large also led to convergence difficulties for the residue vector. Therefore, in the computer program, a small $\gamma$ was initially selected and then $\gamma$ was gradually increased to achieve convergence as well as a good approximation of the sign function.

\section{RESULTS AND DISCUSSION}

\subsection{TUNED SYSTEM}

The frequency response of the tuned system can be obtained by applying the GNR method to equation (6) with $\Delta f_{i}=0$. However, the cyclic symmetry of the stiffness matrix $[K]$ in the tuned case can be exploited to transform the system (6) into $N$ uncoupled equations given by

$$
\Omega^{2} x_{i}^{\prime \prime}+2 \zeta \Omega x_{i}^{\prime}+\left[1+2 R^{2}(1-\cos \theta)\right] x_{i}+\operatorname{Sgn}\left(x_{i}^{\prime}\right)=\rho \cos \left(\tau+\phi_{i}\right), \quad i=1, \ldots, N,
$$

where $\theta=2 \pi n / N$. Several characteristics of the tuned system's response can be observed without solving the equations of motion. First, all component systems have the same amplitude with a cyclic variation of phase, $\theta$, between adjacent component systems. 
Therefore, the tuned system's forced response can be obtained by solving a single nonlinear equation, and there is only one peak in the frequency response. Second, an increase in coupling, $R^{2}$, increases the undamped natural frequencies of the tuned system, and thus shifts the frequency response's peak to a higher excitation frequency. Finally, the ratio $n / N$ can be considered as one parameter, hence, an increase in engine order is equivalent to a decrease in the number of component systems. For $0 \leqslant 2 \pi n / N \leqslant \pi$ the peak of the tuned system's response is shifted to a lower excitation frequency as $n / N$ increases, and vice versa for $\pi \leqslant 2 \pi n / N \leqslant 2 \pi$. The above observations are similar to the results obtained for a linear viscously damped system [7].

Unless otherwise stated, the following results are obtained by the three-harmonic GNR method (i.e., the first, third and fifth harmonic terms are considered), and the amplitudes of vibration are the peak displacements. Figure 2 displays the frequency responses of the tuned system near resonance for various force ratios. One observes that a decrease in $\rho$, which is equivalent to a decrease in the external force or an increase in the amount of dry friction damping, reduces the resonant amplitude of the tuned system. To check the accuracy of the GNR method, a comparison of the steady state displacement waveforms obtained by the GNR method and by numerical integration is performed. Figure 3 shows the time history of the displacement of the first component system for $\rho=3$ and $\Omega=1 \cdot 0$. The result indicates that the one-harmonic approximation is adequate for this excitation frequency and this force ratio. Figure 4 displays the displacement waveforms of the first component system for $\rho=1.5$ and $\Omega=0.6$. It is observed that at such a low excitation frequency the one-harmonic analysis cannot approximate the stick-slip motion well, but that the three-harmonic procedure provides a much better approximation, especially in terms of amplitude and phase. Figure 5 compares the displacement waveforms of the first component system for $\rho=1.28$ and $\Omega=1 \cdot 0$. It is shown that, for this small force ratio such that the non-linear effect due to dry friction damping is dominant, several harmonics are necessary to approximate the motion even at the resonant frequency of the system. However, note that while the three-harmonic analysis results are in much better agreement than the single-harmonic ones with numerical integration in terms of

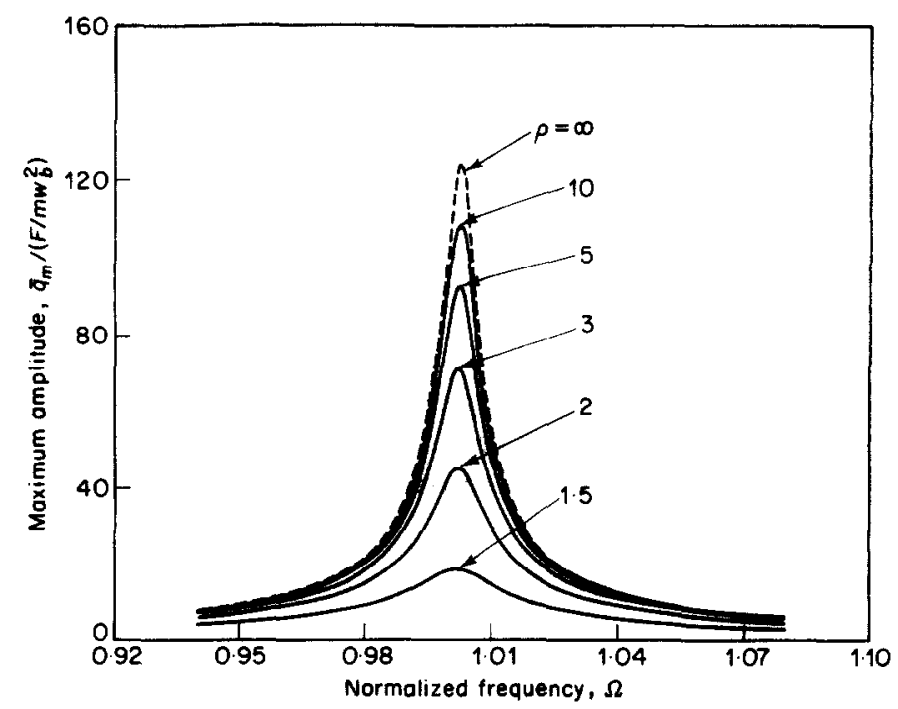

Figure 2. Maximum amplitude of tuned system versus excitation frequency for various force ratios. Tuned system, $R=0 \cdot 1$ (weak coupling), $N=10, n=1, \zeta=0.004, N H=3$. 


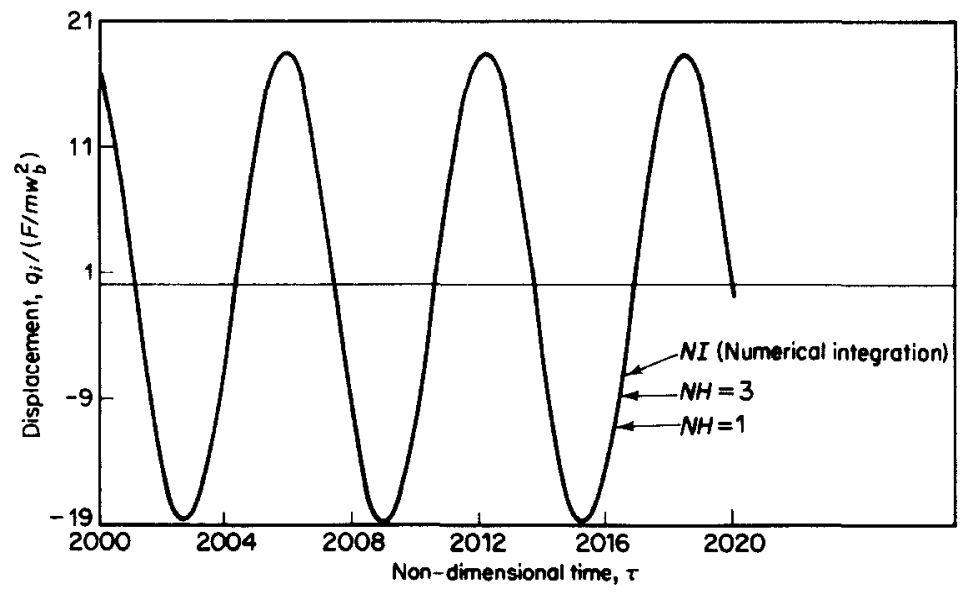

Figure 3. Comparison of the displacement waveforms of the first component system for 1- and 3-harmonic GNR and time integration, for a tuned system, $\rho=3 \cdot 0, \Omega=1 \cdot 0, \zeta=0 \cdot 004 ; R=0.1$ (weak coupling), $N=10, n=1$.

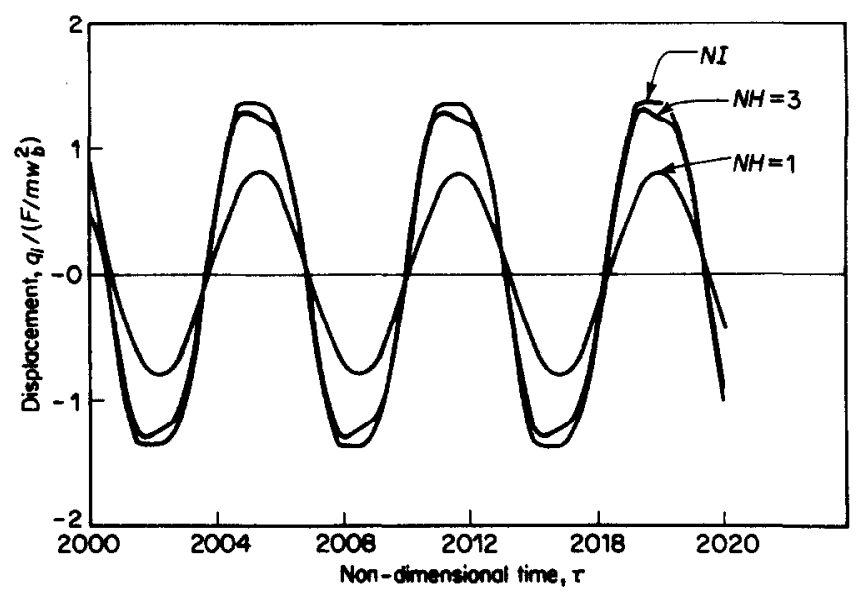

Figure 4. Comparison of the displacement waveforms of the first component system for 1- and 3-harmonic GNR and time integration, for a tuned system, $\rho=1 \cdot 5, \Omega=0 \cdot 6, \zeta=0 \cdot 004$. $R=0 \cdot 1$ (weak coupling), $N=10, n=1$.

both amplitude and phase, more harmonics should be considered to improve further the accuracy of the Galerkin procedure.

These figures suggest that a multi-harmonic analysis should be performed when the nonlinearity is strong or when the excitation frequency is much lower than the resonant frequency. However, although a multi-harmonic analysis provides better accuracy, it increases the computing time significantly and may lead to convergence difficulties for the Newton-Raphson procedure. Hence, the trade-off between accuracy and computing time should be judged by comparing with time integration. When one harmonic cannot provide good accuracy, and multiple harmonics may lead to both convergence problems and larger computing time than time integration, then time integration should be used instead.

\subsection{MISTUNED SYSTEM}

The GNR method was applied to obtain the frequency responses of ten-DOF mistuned systems. The effects of several parameters have been investigated. The calculations for 


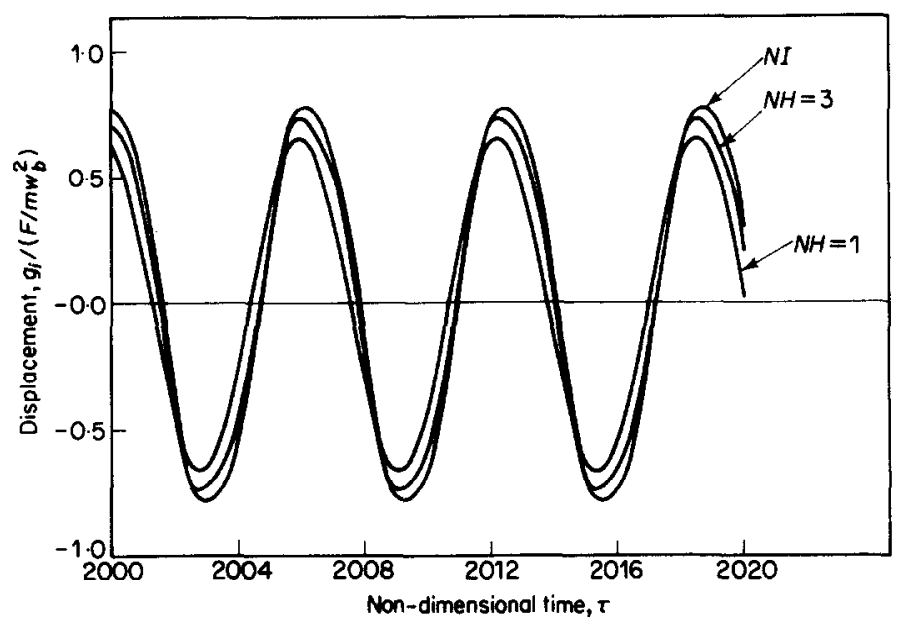

Figure 5. Comparison of the displacement waveforms of the first component system for 1- and 3-harmonic GNR and time integration, for a tuned system, $\rho=1 \cdot 28, \Omega=1 \cdot 0, \zeta=0 \cdot 004$. $R=0 \cdot 1$ (weak coupling), $N=10$, $n=1$.

the mistuned system are deterministic, and the disorder pattern chosen has standard deviation four percent (see Table 1).

The effects of coupling on the forced responses are shown in Figure 6. Figure 6(a) compares the frequency responses of strongly coupled $(R=0.5)$ tuned and mistuned systems. Except for the apparition of several small peaks that are mostly damped out and for the splitting (due to mistuning) of the only peak of the tuned system's response, the frequency response of the strongly coupled mistuned system is similar to that of the tuned system. Figure $6(\mathrm{~b})$ shows that the frequency response of the weakly coupled $(R=0 \cdot 1)$ mistuned system is quite different from that of the tuned system, through the increase of the largest amplitude, the apparition of several peaks of comparable magnitudes, and the significant broadening of the frequency range with high amplitudes. Figure 6 (c) displays the frequency responses of decoupled and weakly coupled mistuned systems. One observes that the response of the weakly coupled mistuned system is qualitatively similar to that of the corresponding decoupled system. In fact, as observed by Wei and

TABLE 1

Mistuning distribution

\begin{tabular}{cc}
\hline Component system no. & Mistuning $\Delta f_{i}$ \\
\hline 1 & 0.06581 \\
2 & 0.03339 \\
3 & -0.03685 \\
4 & -0.00011 \\
5 & -0.05306 \\
6 & -0.00875 \\
7 & 0.04960 \\
8 & -0.04441 \\
9 & 0.00854 \\
10 & -0.01416 \\
Mean & 0.0 \\
Standard deviation & $4 \%$ \\
\hline
\end{tabular}



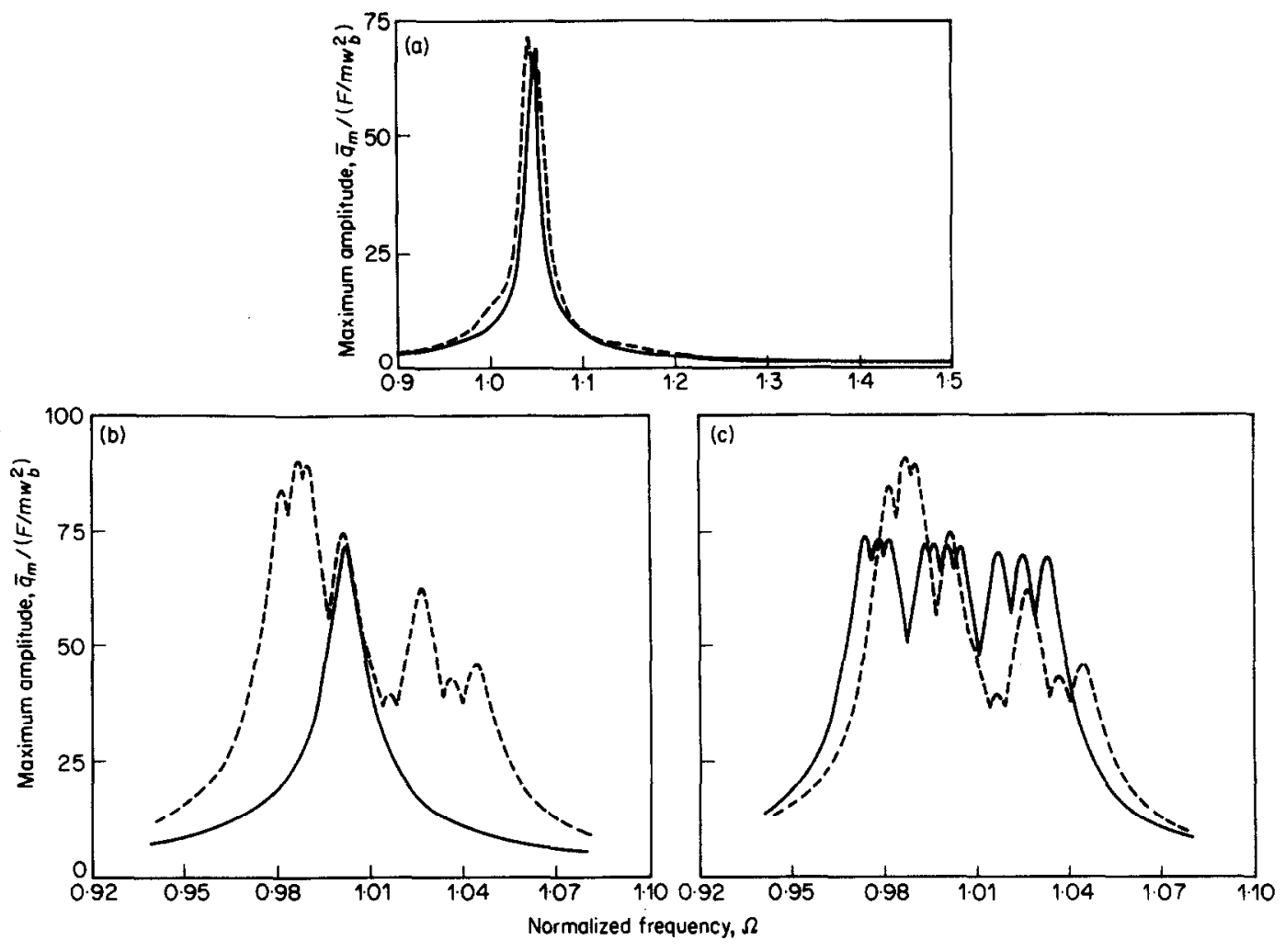

Figure 6. Maximum amplitude versus excitation frequency for $\rho=3 \cdot 0, \zeta=0 \cdot 004$, and various coupling ratios. The mistuning distribution is given in Table $1 . N=10, n=1, N H=3$. (a) shows the frequency responses of the tuned ( $(-)$ and mistuned (- - ) systems for strong coupling $(R=0 \cdot 5)$, while $(b)$ is for weak coupling $(R=0 \cdot 1)$. (c) shows the responses of the decoupled $(-)$ and weakly coupled $(R=0 \cdot 1)$ mistuned $(---)$ systems.

Pierre [7], for a linear viscously damped system the response of the weakly coupled mistuned system is a perturbation of that of the decoupled mistuned system, and the response of the strongly coupled mistuned system is a perturbation of that of the tuned system. This is confirmed here for non-linear dry friction damped systems.

Figure 7 displays the frequency responses of weakly and strongly coupled mistuned systems for various force ratios. For both weak and strong coupling cases, it is observed that a decrease in force ratio (which is equivalent to an increase in dry friction damping or to a decrease in external force) decreases the maximum amplitudes and damps out several small peaks. Also, as the force ratio increases, the response approaches that of the linear viscously damped system.

Figure 8 shows the frequency responses of weakly coupled $(R=0 \cdot 1)$ tuned and mistuned systems for $\rho=1 \cdot 5$. Comparing with Figure 6(b), which is for $\rho=3 \cdot 0$, one observes that for these two different force ratios mistuning has similar effects on the weakly coupled systems through an increase of the largest amplitude and a widening of the passband.

Figure 9 compares the frequency responses of weakly coupled mistuned systems with two types of damping: the first system is viscously damped only $(\zeta=0.02646)$ and the other has both friction $(\rho=1.5)$ and viscous $(\zeta=0.004)$ damping. The damping ratio $(\zeta=0.02646)$ of the first system is selected such that the largest amplitude of the viscously damped tuned system is equal to that of the tuned system with both friction and viscous damping shown in Figure 8. It is observed in Figure 9 that for the equivalent viscously 

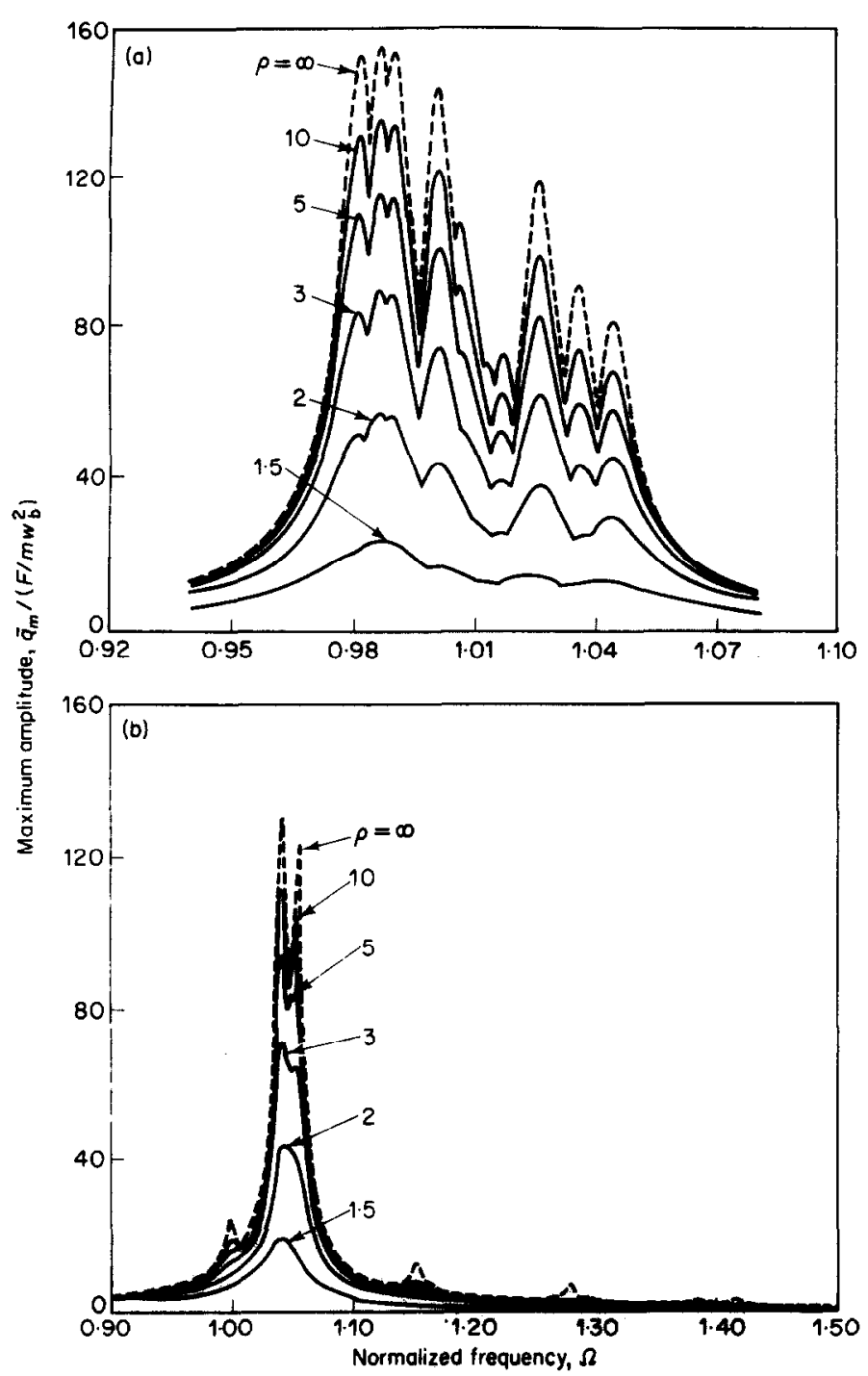

Figure 7. Maximum amplitude of coupled mistuned systems versus excitation frequency, for $\zeta=0.004$ and various force ratios. $N=10, n=1, N H=3$. (a) is for weak coupling $(R=0.1)$, and (b) for strong coupling $(R=0 \cdot 5)$.

damped system the frequency range with high amplitudes is larger, while the increase of the largest amplitude due to mistuning is smaller than that for the system with both friction and viscous damping. These phenomena can be explained by the viscous damping "equivalent" to Coulomb friction damping in a single-DOF system, as follows. Using describing functions, the amount of viscous damping equivalent to friction damping can be shown to be inversely proportional to the product of the vibrational amplitude and the excitation frequency. Hence, the actual equivalent viscous damping in the dry friction damped mistuned system varies with the excitation frequency and the amplitude of each component system. It is worth noting that the equivalent viscous damping selected in Figure 9 is constant and is based on the largest amplitude of the dry friction damped tuned system. However, depending on the product of the excitation frequency and the corresponding amplitude in the dry friction damped mistuned system, the actual equivalent 


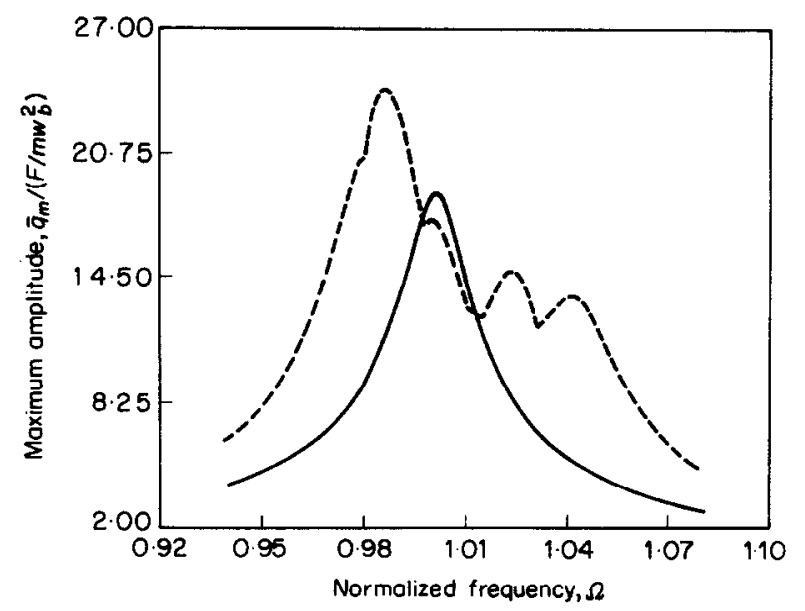

Figure 8. Maximum amplitude versus excitation frequency for weakly coupled tuned ( - ) and mistuned $(--)$ systems $(\rho=1 \cdot 5, \zeta=0 \cdot 004, R=0 \cdot 1, N=10, n=1, N H=3$ ).

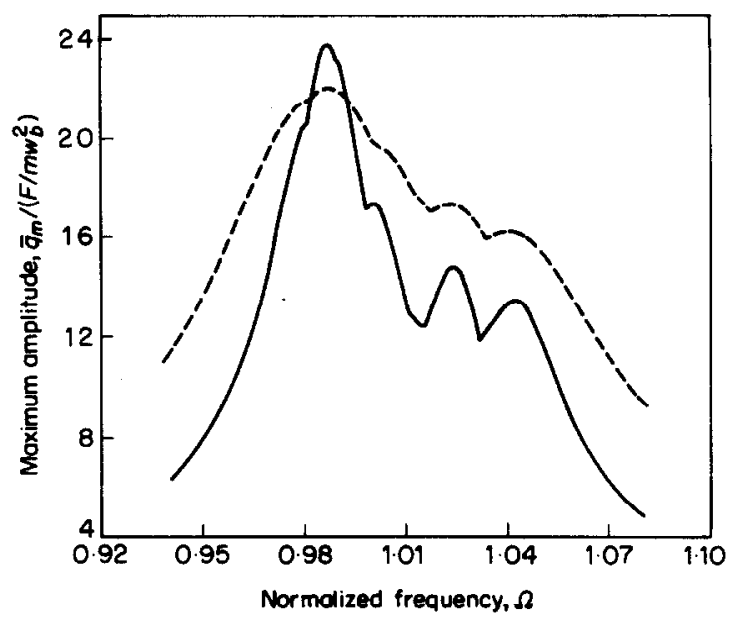

Figure 9. Maximum amplitude versus excitation frequency for weakly coupled $(R=0 \cdot 1)$ mistuned systems with two types of damping: one $(---)$ with only viscous damping $(\zeta=0.02646)$, the other $(-)$ with both friction $(\rho=1.5)$ and viscous $(\zeta=0.004)$ damping. $(N=10, n=1$.)

damping ratio may be larger or smaller. It is observed in Figure 8 that the product of the largest amplitude and the corresponding excitation frequency of the mistuned system is larger than that of the tuned system, therefore the actual equivalent viscous damping of the dry friction damped mistuned system at this excitation frequency is smaller than the selected damping ratio in the viscously damped system, hence leading to a higher largest amplitude for the dry friction damped mistuned system. The conclusion is that dry friction damping appears to be less effective than viscous damping in reducing the increase in largest amplitude caused by mistuning in weakly coupled systems.

\subsection{LOCALIZED FORCED VIBRATIONS}

It has been shown by Wei and Pierre [7] that localized forced vibrations occur in weakly coupled mistuned systems with very small viscous damping. This section is 

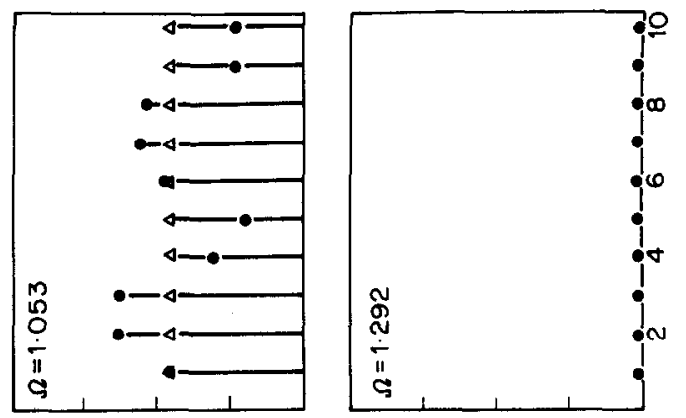

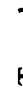

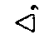

क

2
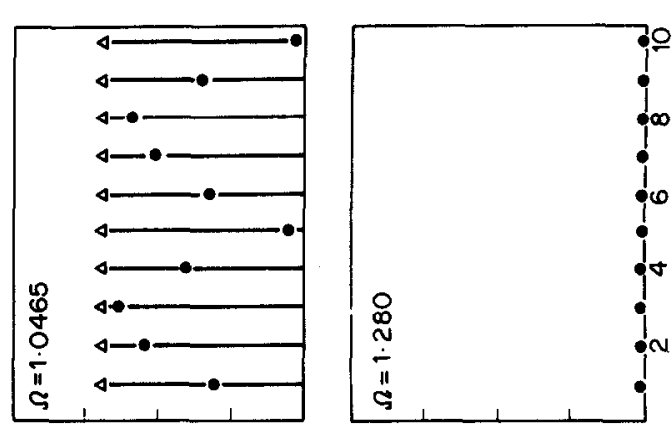

ip
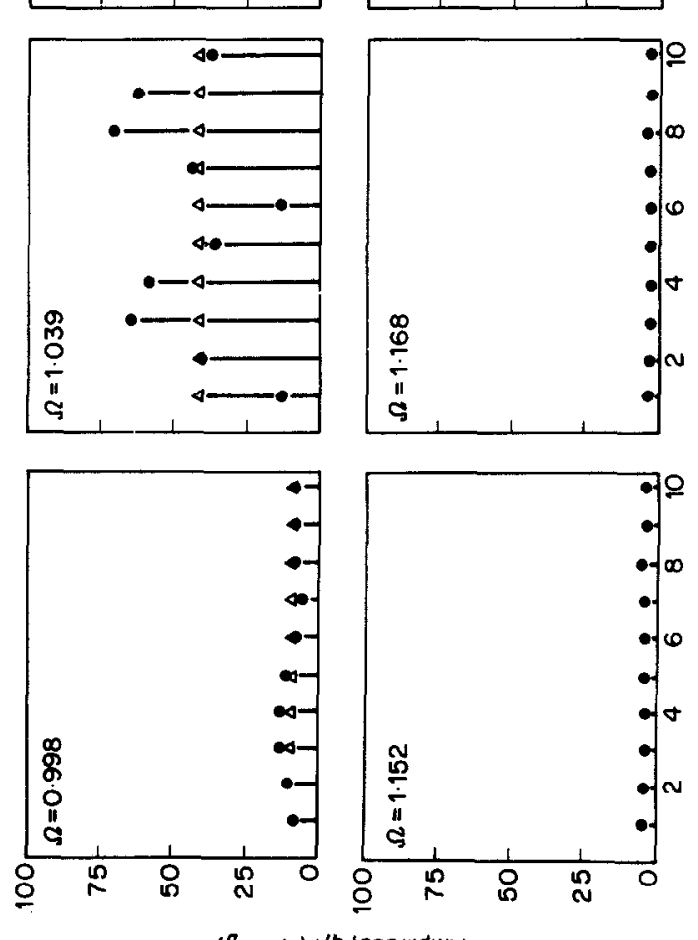

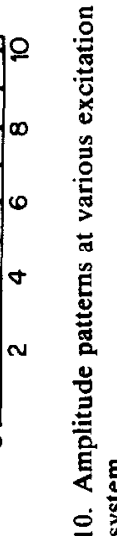

总 
concerned with the effects of viscous damping and dry friction damping on the degree of localization.

Figure 10 shows the amplitude patterns at various excitation frequencies for strongly coupled tuned and mistuned systems with both dry friction damping $(\rho=3 \cdot 0)$ and viscous damping $(\zeta=0.004)$. It is observed that for the mistuned system most component systems experience higher amplitudes at the frequencies close to the resonant frequency of the tuned system, and vibrate with much smaller amplitudes at other excitation frequencies. Also, although some component systems may vibrate with very small amplitudes at frequencies close to the tuned system's resonant frequency, most component systems vibrate with comparable amplitudes; thus no localized vibrations occur. Figure 11 displays the amplitude patterns for weakly coupled tuned and mistuned systems with both dry friction damping $(\rho=3.0)$ and viscous damping $(\zeta=0.004)$. It is shown that at most excitation frequencies the maximum amplitudes of the mistuned system are larger than those of the tuned system except at frequencies very close to the tuned system's resonant frequency. Also, at most frequencies only a few component systems vibrate with much higher amplitudes than others, thus leading to so-called localized forced vibrations. Similar results were observed by Wei and Pierre [7] for a linear viscously damped system. It is also observed that the degree of localization varies with the excitation frequency. The localization phenomenon is most evident at the frequency corresponding to the largest amplitude of the mistuned system, and no strong localization occurs at some frequencies.

Figure 12 shows, for different force ratios, the amplitude patterns at an excitation frequency that leads to a strongly localized behavior for the weakly coupled mistuned system. It is observed that, although the maximum amplitudes decrease significantly as the amount of dry friction damping increases, the degree of localization only slightly decreases with the increase of dry friction damping. Figure 13 shows the amplitude patterns for a weakly coupled mistuned system (with only viscous damping) at the same excitation frequency, for various values of viscous damping. The viscous damping ratios are selected such that the largest amplitudes of the tuned systems are the same as those of viscously damped $(\zeta=0.004)$ systems with dry friction $\rho=4,2$, and 1.5 , respectively. It is observed that, in addition to the decrease in the maximum amplitudes, the degree of localization is also decreased significantly by the increase in viscous damping. This phenomenon can be explained by the fact that an increase in viscous damping decreases the resonant amplitude significantly, but has less effect at other excitation frequencies. Therefore, an increase in viscous damping significantly decreases the amplitudes of the component systems whose resonant frequencies lie close to the excitation frequency, hence leading to a decrease in the degree of localization. Comparing Figure 12(c) with Figure 13(c), one observes that, although the largest amplitudes of the tuned systems are the same (note that the amplitudes of these two tuned systems at this excitation frequency are different), the localized behavior is more pronounced for the dry friction damped mistuned system than for the corresponding viscously damped mistuned system. This can be explained by noting that the viscous damping equivalent to dry friction damping is, at a given excitation frequency, proportional to the inverse of the amplitude, and hence is smaller for a component system of the mistuned assembly with large amplitude and larger for a component system with small amplitude. Therefore, dry friction damping has less effect on the reduction of the maximum amplitude of the mistuned system at a given excitation frequency, and is more susceptible to lead to localized vibrations than viscous damping.

To check the accuracy of the GNR method for mistuned systems, the results can be compared with numerical integration. Figure 14 shows the displacement waveforms of the component systems with maximum and minimum amplitudes at one of the resonant 

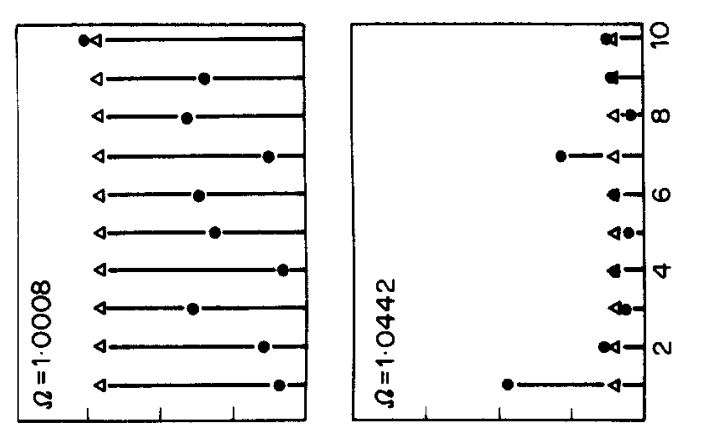

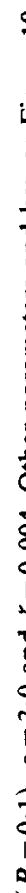
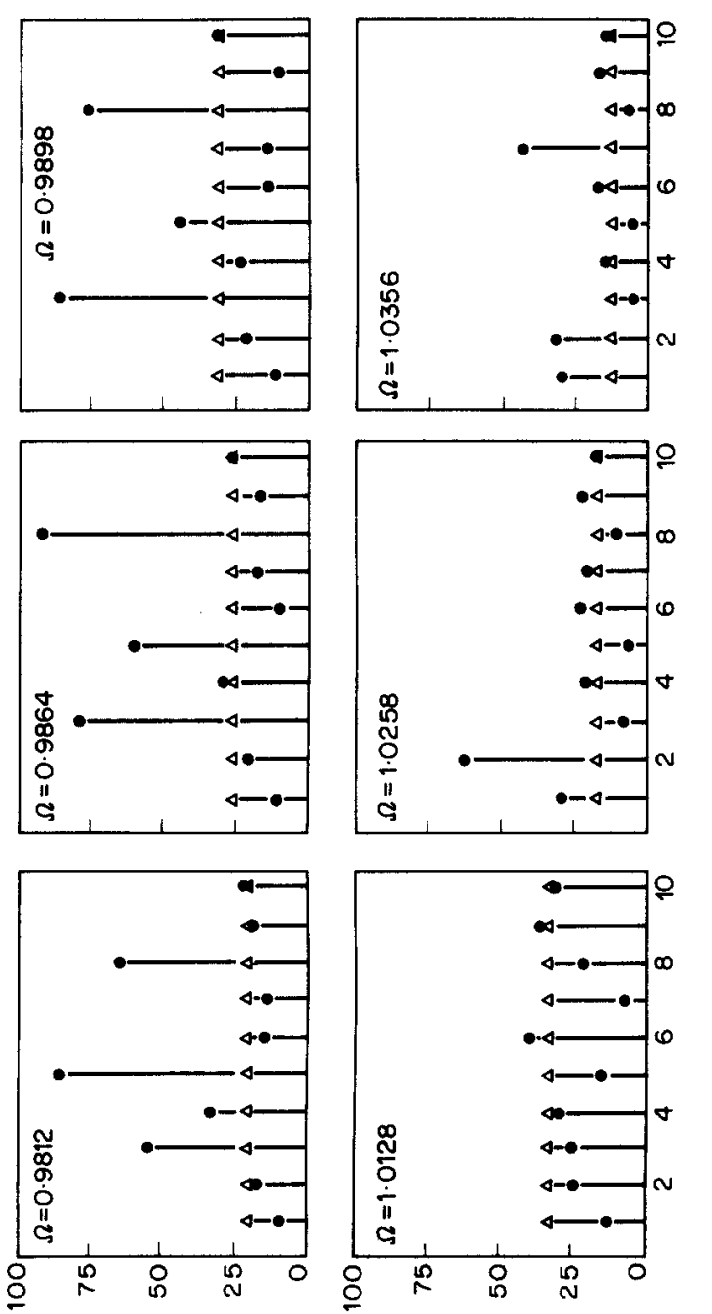

$\left(\frac{a}{2}\right.$ mu/g) $/$ ! 

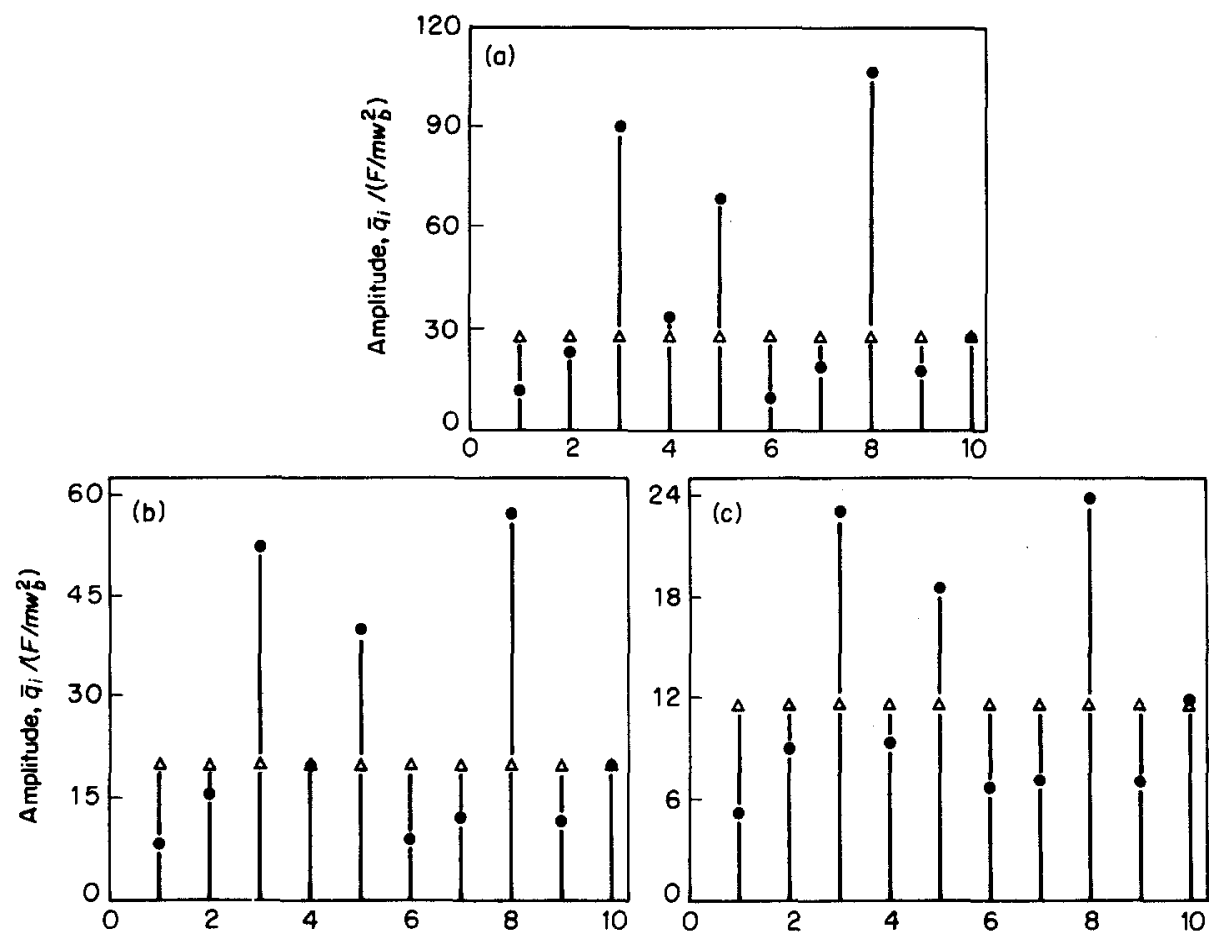

Figure 12. Amplitude patterns at $\Omega=0.9864$ for weak coupling $(R=0 \cdot 1), \zeta=0.004$ and various force ratios $(N=10, n=1, N H=3$ ). (a) is for $\rho=4 \cdot 0$, (b) for $\rho=2 \cdot 0$, and (c) for $\rho=1 \cdot 5$. Key as Figure 10 .
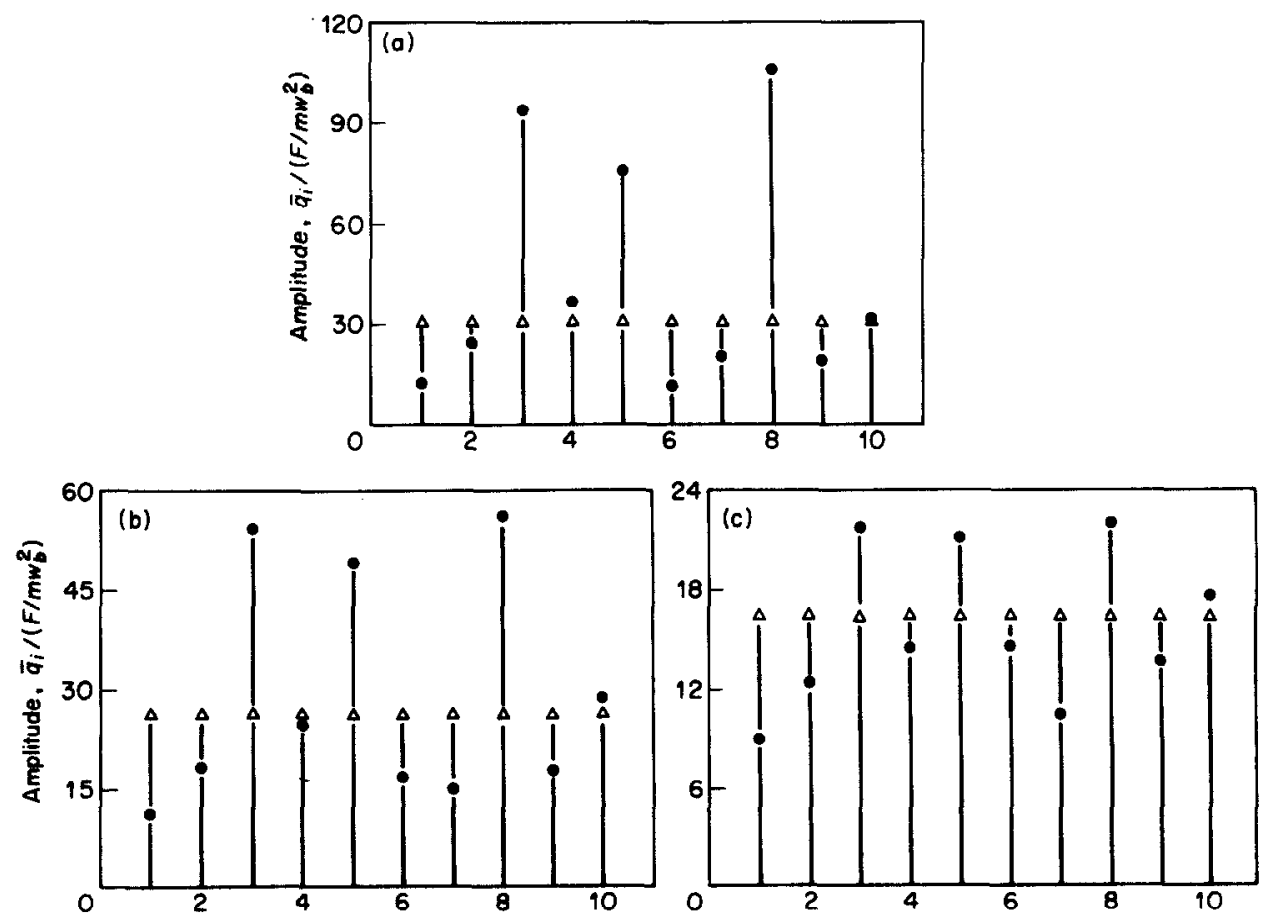

Figure 13. Amplitude patterns at $\Omega=0.9864$ for weak coupling $(R=0.1)$, and various viscous damping ratios ( $N=10, n=1$ ). No dry friction damping is present. (a) is for $\zeta=0.00587$, (b) for $\zeta=0.01101$, and (c) for $\zeta=0.02646$. Key as Figure 10. 

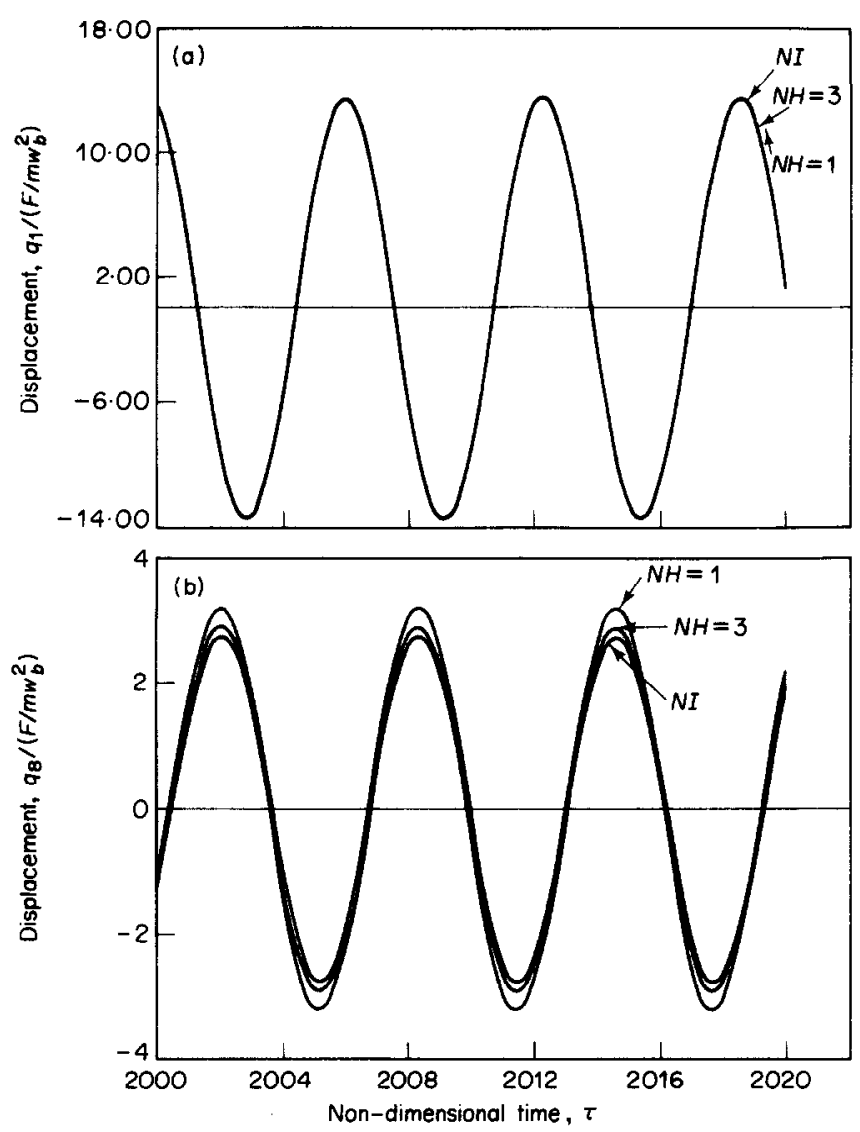

Figure 14. Comparison of the displacement waveforms of the first (a) and eighth (b) component systems of a weakly coupled $(R=0 \cdot 1)$ mistuned system by 1 - and 3-harmonic GNR and time integration, for $\rho=1 \cdot 5$, $\Omega=1.042, \zeta=0.004(N=10, n=1)$.

frequencies of a weakly coupled mistuned system with dry friction damping. It is observed that a one-harmonic approximation gives very good accuracy for the displacement waveform of the component system with the maximum amplitude, but significantly over-predicts the amplitude of the component system with the minimum amplitude. A better approximation is obtained by using three harmonics. It is also interesting to compare the computing cost of obtaining the steady state response by different methods. For the specific case shown in Figure 14, it was found that the computing time of numerical integration is about 5000 times that of the one-harmonic approximation, and that the cost of the three-harmonic analysis is only ten times that of the one-harmonic approximation. Therefore, more harmonics can be used to provide better accuracy with reasonable computing time for the component systems with small amplitudes in weakly coupled mistuned systems.

Another concern about the application of the GNR method is the convergence of the iteration process. It is worth noting that the GNR method cannot be applied to a system for which complete stick motion occurs. However, complete stick motions of some component systems do occur in mistuned systems when dry friction damping is present. In this case time integration has to be used instead. Figure 15 shows the amplitude pattern, obtained by numerical integration, of a strongly coupled mistuned system for $\Omega=1.0485$ 


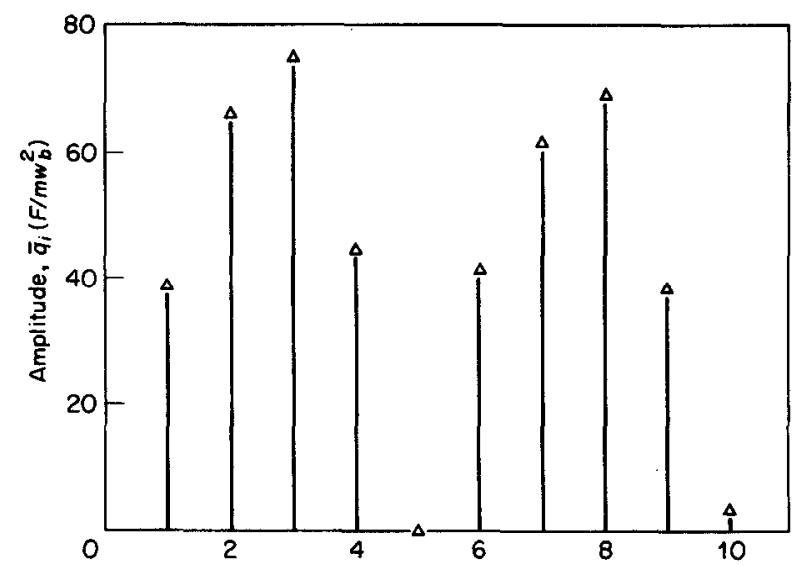

Figure 15. Amplitude pattern at $\Omega=1.0485$ for strong coupling $(R=0.5), \rho=4.0$ and $\zeta=0.004$, by time integration $(N=10, n=1)$.

and $\rho=4 \cdot 0$. It is observed that even for a strongly coupled system the combined effect of mistuning and dry friction damping leads to the complete stick motion of the fifth component system. An alternative approximation of the response by the GNR method may be reached by constraining the component system with stick motion to zero amplitude and modifying the iteration matrices. However, the complete algorithm for this type of motion has not yet been developed. Finally, note that a similar behavior was also observed in the case of localized vibrations of mistuned weakly coupled systems, for which several component systems may remain stuck while others are vibrating with very high amplitudes.

\section{CONCLUSIONS}

The Galerkin/Newton-Raphson method has been applied to obtain the frequency response of a mistuned cyclic structure with many dry friction elements. The following conclusions can be drawn from this study.

1. For a tuned system, all subsystems experience the same amplitude at a given excitation frequency, and the one-harmonic approximation provides good accuracy for small dry friction damping at excitation frequencies close to the resonant frequency of the tuned system. A multi-harmonic analysis is necessary if the system has large friction damping or is excited at frequencies far from the resonant frequency.

2. For a mistuned system, all components may vibrate with very different amplitudes at a given excitation frequency. Although the one-harmonic approximation may generate satisfactory results for the component system with the maximum amplitude, it may not be accurate for the component systems with small amplitudes. Therefore, even at excitation frequencies close to the resonant frequency, and even for systems with small friction damping, a multi-harmonic balance analysis is necessary to obtain an accurate amplitude pattern for the mistuned system.

3. Mistuning has a more significant effect on a weakly coupled system with both dry friction damping and viscous damping than on one with only equivalent viscous damping through the increase of the largest amplitude in the frequency response.

4. Dry friction damping is more susceptible to lead to localized vibrations than viscous damping in a weakly coupled mistuned system, and the degree of localization decreases as damping increases. 


\section{ACKNOWLEDGMENT}

This work was supported by National Science Foundation Grant No. MSM-8700820, Dynamic Systems and Control Program. Dr Elbert L. Marsh is the grant monitor.

\section{REFERENCES}

1. C. H. Hodges 1982 Journal of Sound and Vibration 82, 411-424. Confinement of vibration by structural irregularity.

2. C. H. Hodges and J. WoOdhouse 1983 Journal of the Acoustical Society of America 74, 894-905. Vibration isolation from irregularity in a nearly periodic structure: theory and measurements.

3. C. PIERRE and E. H. Dowell 1987 Journal of Sound and Vibration 114, 549-564. Localization of vibrations by structural irregularity.

4. C. PIERre 1987 AIAA Paper 87-0774, Proceedings of the 28th AIAA/ASME/ASCE/AHS Structures, Structural Dynamics and Materials Conference, Monterey, California. Localized free and forced vibrations of nearly periodic disordered structures.

5. N. A. VALERO and O. O. BENDIKSEN 1986 American Society of Mechanical Engineers Journal of Engineering for Gas Turbines and Power 108(2), 293-299. Vibration characteristics of mistuned shrouded blade assemblies.

6. S.-T. WEI and C. PIERRE 1988 American Society of Mechanical Engineers Journal of Vibration, Acoustics, Stress, and Rellability in Design 110(4), 429-438. Localization phenomena in mistuned assemblies with cyclic symmetry, Part I: free vibrations.

7. S.-T. WEI and C. PIERRE 1988 American Society of Mechanical Engineers Journal of Vibration, Acoustics, Stress, and Reliability in Design 110(4), 439-449. Localization phenomena in mistuned assemblies with cyclic symmetry, Part II: forced vibrations.

8. O. O. BENDIKSEN 1987 American Institute of Aeronautics and Astronautics Journal 25(9), 1241-1248. Mode localization phenomena in large space structures.

9. G. J. KISSEL 1987 AIAA Paper 87-0819, Proceedings of the 28th AIAA/ASME/ASCE/AHS Structures, Structural Dynamics and Materials Conference, Monterey, California. Localization in disordered periodic structures.

10. S. L. LAU, Y. K. CheUNG and S. Y. WU 1982 American Society of Mechanical Engineers Journal of Applied Mechanics 49, 849-853. A variable parameter incremental method for dynamic instability of linear and nonlinear elastic systems.

11. C. PIERRE and E. H. DOWELl 1985 American Society of Mechanical Engineers Journal of Applied Mechanics 52(3), 693-697. A study of dynamic instability of plates by an extended incremental harmonic balance method.

12. C. PierRe, A. A. FerRI and E. H. Dowell 1985 American Society of Mechanical Engineers Journal of Applied Mechanics 52(4), 958-964. Multi-harmonic analysis of dry friction damped systems using an incremental harmonic balance method.

13. A. A. FERRI and E. H. DOWELL 1985 Proceedings of the Fifth VPI\&SU/AIAA Symposium on Dynamics and Control of Large Structures, Blacksburg, Virginia. Frequency domain solutions to multi-degree-of-freedom, dry friction damped systems under periodic excitation.

14. A. A. FERRI 1986 American Society of Mechanical Engineers Journal of Applied Mechanics 53(2), 455-457. On the equivalence of the incremental harmonic balance method and the harmonic balance-Newton Raphson method.

15. F. H. LING and X. X. WU 1987 International Journal of Non-Linear Mechanics 22(2), 89-98. Fast Galerkin method and its application to determine periodic solutions of non-linear oscillators.

16. D. I. G. Jones and A. Muszyńska 1983 Vibrations of Bladed Disk Assemblies, Proceedings of the Ninth Biennial Conference on Mechanical Vibration and Noise, Dearborn, Michigan. A discrete model of a multiple blade system with interblade slip.

17. A. MUSZYŃSKA, D. I. G. JONES, T. LAGNESE and L. WHITFORD 1981 Shock and Vibration Bulletin 51(3), 89-110. On non-linear response of multiple blade systems.

18. A. MUSZYŃSKA and D. I. G. JONES 1983 American Society of Mechanical Engineers Journal of Vibration, Acoustics, Stress, and Reliability in Design 105, 434-443. A parametric study of dynamic response of a discrete model of turbomachinery bladed disk.

19. J. H. GRIFFIN 1980 American Society of Mechanical Engineers Joumal of Engineering for Power 102, 329-333. Friction damping of resonant stresses in gas turbine engine airfolls. 
20. J. H. GRIFFIN and A. SINHA 1985 American Society of Mechanical Engineers Journal of Engineering for Gas Turbines and Power 107, 205-211. The interaction between mistuning and friction in the forced response of bladed disk assemblies.

21. A. SINHA and J. H. GRIFFIN 1985 Journal of Sound and Vibration 103, 341-356. Stability of limit cycles in frictionally damped and aerodynamically unstable rotor stages.

22. T. M. CAMERON, J. H. GRIFFIN, R. E. KIELB and T. M. HoosAC 1987 The Role of Damping in Vibration and Noise Control, Proceedings of the 11 th Biennial Conference on Mechanical Vibration and Noise, Boston, Massachusetts. An integrated approach for friction damper design.

$$
\begin{gathered}
\text { APPENDIX I } \\
\mathbf{R}\left(\mathbf{y}_{0}\right)=[C L] \mathbf{y}_{0}+\mathbf{S}\left(\mathbf{y}_{0}\right)
\end{gathered}
$$

and

$$
\begin{aligned}
& \partial \mathbf{R} /\left.\partial \mathbf{y}\right|_{0}=[C L]+\partial \mathbf{S} /\left.\partial \mathbf{y}\right|_{0}, \\
& {[C L]=\left[\begin{array}{ccccc}
{\left[L_{11}\right]} & {\left[L_{12}\right]} & \ldots & \ldots & {\left[L_{1 N}\right]} \\
{\left[L_{21}\right]} & {\left[L_{22}\right]} & \ldots & \ldots & {\left[L_{2 N}\right]} \\
\cdots & \cdots & \ldots & \ldots & \cdots \\
\ldots & \ldots & \ldots & \ldots & \ldots \\
{\left[L_{N 1}\right]} & {\left[L_{N 2}\right]} & \cdots & \cdots & {\left[L_{N N}\right]}
\end{array}\right],} \\
& {\left[L_{\alpha \beta}\right]=\left[\begin{array}{cc}
{\left[A_{\alpha \beta}\right]} & {\left[B_{\alpha \beta}\right]} \\
-\left[B_{\alpha \beta}\right] & {\left[A_{\alpha \beta}\right]}
\end{array}\right], \quad \alpha, \beta=1, \ldots, N,} \\
& {\left[A_{\alpha \beta}\right]_{i j}=\left(-\Omega^{2} l^{2} m_{\alpha \beta}+k_{\alpha \beta}\right) \delta_{i}^{j}, \quad i, j=1, \ldots, N H,} \\
& {\left[B_{\alpha \beta}\right]_{i j}=\left(\Omega l c_{\alpha \beta}\right) \delta_{i}^{j}, \quad i, j=1, \ldots, N H \text {, }}
\end{aligned}
$$

where $l=2 j-1$ in equations (A5) and (A6). The terms $m_{\alpha \beta}, c_{\alpha \beta}$ and $k_{\alpha \beta}$ are the elements of $[M],[C]$ and $[K]$, respectively.

$$
\begin{aligned}
& \partial \mathbf{S} /\left.\partial \mathbf{y}\right|_{0}=\left[\begin{array}{ccccc}
{\left[H_{11}\right]} & {[0]} & \ldots & \ldots & {[0]} \\
{[0]} & {\left[H_{22}\right]} & {[0]} & \ldots & \ldots \\
\ldots & \ldots & \ldots & \ldots & \ldots \\
\ldots & \ldots & \ldots & \ldots & \ldots \\
{[0]} & \ldots & \ldots & {[0]} & {\left[H_{N N}\right]}
\end{array}\right], \\
& {\left[H_{\alpha \alpha}\right]=\left[\begin{array}{ll}
{\left[D_{\alpha \alpha}^{11}\right]} & {\left[D_{\alpha \alpha}^{12}\right]} \\
{\left[D_{\alpha \alpha}^{21}\right]} & {\left[D_{\alpha \alpha}^{22}\right]}
\end{array}\right], \quad \alpha=1, \ldots, N,} \\
& {\left[D_{\alpha \alpha}^{11}\right]_{i j}=-\left.\frac{1}{\pi} \int_{0}^{2 \pi} \frac{\partial \operatorname{Sgn}\left(x_{\alpha}^{\prime}\right)}{\partial x_{\alpha}^{\prime}}\right|_{0} l \sin l \tau \cos n \tau \mathrm{d} \tau, \quad i, j=1, \ldots, N H \text {, }} \\
& {\left[D_{\alpha \alpha}^{12}\right]_{i j}=\left.\frac{1}{\pi} \int_{0}^{2 \pi} \frac{\partial \operatorname{Sgn}\left(x_{\alpha}^{\prime}\right)}{\partial x_{\alpha}^{\prime}}\right|_{0} l \cos l \tau \cos n \tau \mathrm{d} \tau, \quad i, j=1, \ldots, N H,} \\
& {\left[D_{\alpha \alpha}^{21}\right]_{i j}=-\left.\frac{1}{\pi} \int_{0}^{2 \pi} \frac{\partial \operatorname{Sgn}\left(x_{\alpha}^{\prime}\right)}{\partial x_{\alpha}^{\prime}}\right|_{0} l \cos l \tau \sin n \tau \mathrm{d} \tau, \quad i, j=1, \ldots, N H,} \\
& {\left[D_{\alpha \alpha}^{11}\right]_{i j}=\left.\frac{1}{\pi} \int_{0}^{2 \pi} \frac{\partial \operatorname{Sgn}\left(x_{\alpha}^{\prime}\right)}{\partial x_{\alpha}^{\prime}}\right|_{0} l \sin l \tau \sin n \tau \mathrm{d} \tau, \quad i, j=1, \ldots, N H,}
\end{aligned}
$$

where $l=2 j-1$ and $n=2 i-1$ in equations (A9)-(A12).

$$
\begin{array}{r}
\mathbf{S}\left(\mathbf{y}_{0}\right)=\left\{T_{1}^{c 1}, \ldots, T_{1}^{c(2 N H-1)}, T_{1}^{s 1}, \ldots, T_{1}^{s(2 N H-1)}, T_{2}^{c 1}, \ldots, \ldots, T_{N}^{s(2 N H-1)}\right\}^{\mathrm{T}}, \quad \text { (A13) } \\
T_{i}^{c l}=\left(-\rho \cos \phi_{i}\right) \delta_{l}^{1}+\frac{1}{\pi} \int_{0}^{2 \pi} \operatorname{Sgn}\left(x_{i}^{\prime}\right) \cos l \tau \mathrm{d} \tau, \quad i=1, \ldots, N, \quad l=1,3, \ldots,(2 N H-1),
\end{array}
$$




$$
T_{i}^{s l}=\left(\rho \sin \phi_{i}\right) \delta_{l}^{1}+\frac{1}{\pi} \int_{0}^{2 \pi} \operatorname{Sgn}\left(x_{i}^{\prime}\right) \sin l \tau \mathrm{d} \tau, \quad i=1, \ldots, N, \quad l=1,3, \ldots,(2 N H-1) .
$$

Note that $\operatorname{Sgn}\left(x_{i}^{\prime}\right)$ is either the exact sign function or the approximate expression (15). When the exact sign function is used, $\partial \operatorname{Sgn}\left(x_{i}^{\prime}\right) / \partial x_{i}^{\prime}$ is twice the Dirac delta function $\delta\left(x_{i}^{\prime}\right)$. The integrals involving the sign function and the delta function can be computed in closed form by the method described in Pierre et al. [12]. If the approximate expression is chosen, then the derivative is obtained as

$$
\frac{\partial \operatorname{Sgn}\left(x_{i}^{\prime}\right)}{\partial x_{i}^{\prime}} \simeq \frac{2}{\pi} \frac{\gamma}{1+\left(y x_{i}^{\prime}\right)^{2}}
$$

and the integrals comprising the approximate sign function and its derivative can be computed by numerical integration.

\section{APPENDIX II: NOMENCLATURE}

$b \quad$ viscous damping coefficient

[C] viscous damping matrix

$F$ external force amplitude

$F_{i} \quad$ external force on the $i$ th component system

$F_{d} \quad$ dry friction force

[I] identity matrix

$k_{b} \quad$ nominal component system's stiffness

$k_{b i} \quad i$ th component system's stiffness

$k_{c} \quad$ coupling stiffness

$[K]$ stiffness matrix

$m_{i}=m$, mass of $i$ th component system

[M] mass matrix

$n$ engine order excitation

$N$ number of component systems

$\mathrm{NH}$ number of harmonics

$q_{i} \quad$ displacement of $i$ th component system

$\bar{q}_{i} \quad$ displacement amplitude of $i$ th component system

$\bar{q}_{m} \quad$ maximum displacement amplitude throughout the assembly at a given excitation frequency

$R^{2}=\omega_{c}^{2} / \omega_{h}^{2}$, dimensionless coupling

$\mathbf{R}$ residue vector

Sgn $\operatorname{sign}$ function, $\operatorname{Sgn}(x)=-1$ for $x<0,=0$ for $x=0$, and $=1$ for $x>0$

$t$ time

$x_{i} \quad=m \omega_{b}^{2} q_{i} / F_{d}$, non-dimensional displacement

$\delta_{i}^{j} \quad$ Kronecker symbol, $=1$ for $i=j$, and $=0$ for $i \neq j$,

$\Delta f_{t}=\left(\omega_{b i}^{2}-\omega_{b}^{2}\right) / \omega_{b}^{2}$, mistuning of $i$ th component system

$\omega \quad$ excitation frequency

$\omega_{b} \quad=\sqrt{k_{b} / m}$, nominal component system's natural frequency

$\omega_{b i} \quad=\sqrt{k_{b i} / m}$, natural frequency of $i$ th component system

$\omega_{\mathrm{c}} \quad=\sqrt{k_{c} / m}$, coupling frequency

$\Omega \quad=\omega / \omega_{b}$, dimensionless excitation frequency

$\phi_{i} \quad$ phase angle of $i$ th force component

$\rho=F / F_{d}$, dimensionless force ratio

$\tau=\omega t$, non-dimensional time

$\theta \quad=2 \pi n / N$

$\zeta \quad=b /\left(2 \sqrt{k_{b} m}\right)$, viscous damping ratio

$\mathrm{T}$ superscript, denotes a transpose

$(\cdot)^{\prime} \quad \equiv \mathrm{d}(\cdot) / \mathrm{d} \tau$

$(\cdot) \quad \Rightarrow \mathrm{d}(\cdot) / \mathrm{d} t$

f boldface symbol denotes a vector 